\title{
Low total gamma globulin level discovery at diffuse large B cell lymphoma diagnosis predicts high risk of infection-related death: data from a monocentric retrospective study
}

\author{
Alexandre NGUYEN ${ }^{1}$, Nicolas Martin-Silva ${ }^{1}$, Hubert de Boysson ${ }^{1}$, Samuel Deshayes ${ }^{1}$, \\ Anne-Claire Gac ${ }^{2}$, Emilie Reboursière ${ }^{2}$, Ghandi Damaj ${ }^{2}$, and Achille Aouba ${ }^{1}$ \\ ${ }^{1} \mathrm{CHU}$ Caen \\ ${ }^{2} \mathrm{CHU}$ Caen Institut d'Hématologie de Basse-Normandie
}

April 15, 2021

\begin{abstract}
Objective: Diffuse-large-B-cell-lymphoma (DLBCL) can complicate B-cell-primary-immunodeficiencies (PIDs) course or induce total gamma-globulin level (TGL) lowering, whose clinical status as an effective secondary immunodeficiency (SID) remains unspecified. This study aims to assess the frequency, clinical and prognostic relevance of the lowest TGLs discovered at DLBCL diagnosis. Results: In a two year monocentric retrospective cohort, 96 patients diagnosed with DLBCL who had a serum electrophoresis (SEP) were included. Patients were divided into the lowest (L)- and the highest (H)-TGLs (TGL [?]5.5 g/L and TGL > $5.5 \mathrm{~g} / \mathrm{L})$ subgroups and compared for outcomes, including fatal infectious events. In our cohort, 12 (12.5\%; 8 males; median age: 68 [55-82] years) exhibited L-TGL. There was no differences regarding demographics, Ann-Arbor-lymphomastages, inflammatory parameters or chemotherapy regimen between both groups. However, overall $(10 / 12,83.3 \%$ versus $22 / 96$, $26.2 \% ; \mathrm{p}=0.03)$ and infection-related death rates $(10 / 12,83 \%$ versus $6 / 96,6.2 \% ; \mathrm{p}<0.001)$ were significantly higher in the L-TGL group. Conclusion: We demonstrate for the first time the strong negative impact of L-TGL on overall and infectionrelated mortality in DLBCL. Prospective studies should distinguish DLBCL-related SIDs from preexisting humoral PIDs, using biomolecular testing and post-treatment TGLs monitoring to determine the best management strategy for infectious risk during DLBCL treatment in L-TGL context.
\end{abstract}

Low total gamma globulin level discovery at diffuse large B cell lymphoma diagnosis predicts high risk of infection-related death: data from a monocentric retrospective study

Short title : Ig level predicts deaths in B lymphoma

Alexandre Nguyen ${ }^{1 *}$, Nicolas Martin-Silva ${ }^{1}$, Hubert de Boysson ${ }^{1}$, Samuel Deshayes ${ }^{1}$, Anne-Claire Gac $^{2}$, Emilie Reboursière ${ }^{2}$, Gandhi Damaj ${ }^{2}$ and Achille Aouba ${ }^{1}$

${ }^{1}$ Department of Internal Medicine and Clinical Immunology, Normandie Univ, UNICAEN, CHU de Caen Normandie, Caen, France

${ }^{2}$ Department of Clinical Haematology, Normandie Univ, UNICAEN, CHU de Caen Normandie, Caen, France

* These authors contributed equally to this work

Corresponding author : Nguyen Alexandre, Email: nguyen-a@chu-caen.fr, Department of Internal Medicine and Clinical Immunology, CHU Côte de Nacre - Université Basse Normandie, Avenue de la Côte de Nacre, 14000 CAEN, France, Tel: +3323106 45 79, Fax: +33231064954

Abstract word count : 200, Manuscript word count: 2672 
Number of references: 25, Number of tables: 3,Number of figures: 1

Number of supplemental files: 0

Declarations

Ethics approval and consent

Approval of the Research Ethics Committee (CLERS) of Caen (ref: 03/2020/AOU)

\section{Consent for publication}

Patients gave consent for collections of data regarding their medical condition.

\section{Availability of data and materials}

The datasets analysed during the current study are available from Pr Gandhi Damaj on reasonable request.

\section{Conflict of interest}

The authors declare that they have no conflict of interest.

\section{Funding}

This study received no specific fundings for research

\section{Authors' contributions}

Alexandre Nguyen and Achille Aouba: Performed the research and wrote the paper

Nicolas Martin-Silva: Designed the research study

Hubert de Boysson, Samuel Deshayes: performed statistical analyses

Anne-Claire Gac, Emilie Reboursière, Gandhi Damaj: included patients into registry

Acknowledgements (Not applicable)

\section{Abstract}

Objective: Diffuse-large-B-cell-lymphoma (DLBCL) can complicate B-cell-primary-immunodeficiencies (PIDs) course or induce total gamma-globulin level (TGL) lowering, whose clinical status as an effective secondary immunodeficiency (SID) remains unspecified. This study aims to assess the frequency, clinical and prognostic relevance of the lowest TGLs discovered at DLBCL diagnosis.

Results: In a two year monocentric retrospective cohort, 96 patients diagnosed with DLBCL who had a serum electrophoresis (SEP) were included. Patients were divided into the lowest (L)- and the highest (H)-TGLs (TGL [?]5.5 g/L and TGL >5.5 g/L) subgroups and compared for outcomes, including fatal infectious events. In our cohort, 12 (12.5\%; 8 males; median age: 68 [55-82] years) exhibited L-TGL. There was no differences regarding demographics, Ann-Arbor-lymphoma-stages, inflammatory parameters or chemotherapy regimen between both groups. However, overall $(10 / 12,83.3 \%$ versus $22 / 96,26.2 \% ; p=0.03)$ and infection-related death rates $(10 / 12,83 \%$ versus $6 / 96,6.2 \% ; \mathrm{p}<0.001)$ were significantly higher in the L-TGL group.

Conclusion: We demonstrate for the first time the strong negative impact of L-TGL on overall and infectionrelated mortality in DLBCL. Prospective studies should distinguish DLBCL-related SIDs from preexisting humoral PIDs, using biomolecular testing and post-treatment TGLs monitoring to determine the best management strategy for infectious risk during DLBCL treatment in L-TGL context.

KEY WORDS: Tumour Immunology, Immunodeficiency Diseases, Cancer, Antibodies, Immunodeficiency Diseases

\section{Introduction}


Recurrent bacterial, viral, fungal and/or parasitic infections are the hallmark events of primary and secondary immunodeficiencies. The most common forms of primary immunodeficiencies (PIDs) are related to inherited adaptive immune system dysfunctions that involve B and/or T cells not unfrequently discovered in adulthood. For secondary immunodeficiencies (SIDs), the main causes correspond to chemotherapy or immunosuppressant use or are related to underlying diseases such as cancers, lymphomas, and systemic inflammatory/autoimmune diseases. Other causes and mechanisms of SIDs proceed from protein wasting or metabolic disorders, including severe general state impairment.

In addition to infections, other events, such as autoimmune cytopenias, cancers or lymphoproliferative disorders, including diffuse large B cell lymphoma (DLBCL), can complicate the natural history of PIDs.(1-3) The most frequent subtype, common variable immunodeficiency (CVID), renders affected patients more prone to developing lymphomas(4-7) and mostly frequent infections identified as the main feature, responsible for diagnosis and main cause of death in this population. $(2,8,9)$ The diagnosis of PIDs is often delayed for several years, because of their clinical heterogeneity and the higher frequency of banal and neglected infections. In this context, both the onset date and natural history of the different complications of PIDs, such as malignant events, remain little known.

We therefore hypothesized that DLBCL with a datable diagnosis might also reveal PIDs with possible previous infectious events that had not led to the recognition of a PID. No study has yet determined the prevalence of PIDs in adults revealed by lymphoma since the diagnostic criteria of PIDs do not take into account lymphoma.(10-15) On the one hand, humoral PIDs, including CVID, are frequent forms of PIDs and can be easily be suggested by low serum total gamma-globulin level identified using serum electrophoresis (SEP) performed at DLBCL diagnosis. On the other hand, DLBCL, the most frequent of lymphoproliferative disorders, can also induce a decrease in serum total gamma-globulin level (TGL) because of clonal selection and proliferation. However, the frequency, the risk and prognosis of infectious events related to L-TGL discovered at DLBCL diagnosis are unknown and no specific therapeutic strategy is therefore recommended in this context. Meanwhile, in the context of multiple myeloma or chronic lymphoid leukemia, the lowest (L)-TGL are well recognized to correspond to an SID with a documented increased infectious events and risk that therefore requires polyvalent immunoglobulin infusions.(16-19)

The clinical relevance and utility of SEP performance for L-TGL research in DLBCL is unknown, both at diagnosis and during the therapeutic follow-up of the lymphoproliferative disorder. Moreover, the association of SEP and DLBCL is of particular interest, as SEP can be easily and routinely performed at DLBCL diagnosis, in order to at least assess the functionality of the B cell compartment and because DLBCL onset, unlike the onset of indolent lymphomas, can easily be dated based on B symptoms and rapid tumoral syndrome.

Herein, we conducted a retrospective study to assess the clinical and prognostic relevance of L-TGL discovery at DLBCL diagnosis in Caen Tertiary Hospital. Our main judgment criteria were the frequency of L-TGL in comparison to H-TGL at DLBCL diagnosis, the mortality rates and main causes of death, especially those related to infectious events among subgroups.

\section{Methods}

\section{DLBCL patients 'cohort}

\section{Inclusion criteria and definitions}

We retrospectively extracted from the monocentric lymphoma database of Caen University Hospital all patients diagnosed with DLBCL between January 2015 and December 2016, counted those with SEP performed at diagnosis before receiving any specific treatments. Included patients were categorized into two groups according to whether they exhibited L-TGL or H-TGL on SEP at DLBCL diagnosis. Although CVID diagnosis requires a threshold $<5 \mathrm{~g} / \mathrm{L}$ of IgG isotype in addition to other biological, clinical and anamnestic criteria(20-22), we chose serum total gamma-globulin level because it is an easily available biological test. 
We defined L-TGL as all TGL values [?] $5.5 \mathrm{~g} / \mathrm{L}$ on SEP as it is $<50 \%$ of lower range of our laboratory norm similarly to what was used in others studies in hematological malignancies(19) and because IgG represent $75 \%$ of TGL in the absence of monoclonal gammapathy, it would be unlikely to have more than $5 \mathrm{~g} / \mathrm{L}$ of $\operatorname{IgG}$.

\section{Exclusion criteria}

As our hypothesis is based on L-TGL being a possible marker of PID or SID related to the DLBCL condition, we excluded all patients exhibiting an associated monoclonal peak on SEP; unrelated increased loss of serum protein such as exudative enteropathy, extended burns or nephrotic syndrome; previous treatment with cytotoxic drugs or immunosuppressant; and previous PID or SID diagnosed before DLBCL.

\section{Studied parameters}

For all patients, demographic information at diagnosis, disease status, chemotherapy regimen, Ann Arbor staging classification, central nervous system involvement (CNS) involvement, and international prognosis index (IPI) and outcome data were collected until the last follow-up date. For biological data, C-reactive protein (CRP), albumin, lymphocytes count, lactate deshydrogenase (LDH) and ferritin levels were collected at time of diagnosis in the absence of an infectious process, and when available, $\operatorname{IgG}, \operatorname{IgM}$, and $\operatorname{IgA}$ levels were implemented. We considered the albuminemia/gammaglobulinemia ratio (AG ratio) to discriminate possible causes for L-TGL : 1) For an unspecific decrease in TGL related to general state impairment, a decrease in albumin should be associated to decrease in TGL, with an unaltered AG ratio; 2) For a specific decrease in TGL directly related to our hypotheses, i.e., L-TGL due to either an undiagnosed PID or a SID developed with DLBCL, the albumin level should be close to normal value, resulting in an increase in the AG ratio.

\section{Statistical analyses}

Data were compiled as the median [range] or a number (\%). Continuous variables were analyzed using the Mann-Whitney test, and Fisher's test was used to compare categorical variables. A p value $<0.05$ was considered statistically significant.

Predictors of death in DLBCL were evaluated using COX proportional hazard models. All the variables showing a univariate p-value less than 0.20 were entered into a multivariable logistic regression model. Results were expressed as hazard ratios (HRs) with 95\% confidence intervals (95\% CI). Two-sided P-values lower than 0.05 were considered significant.

Overall survival in DLBCL and subgroups L-TGL and H-TGL were analyzed using the Kaplan-Meier method, and these variables were compared using the log-rank test.

All statistical analyses were performed using GraphPad Prism software (7.0) and JMP 9.0.1 (SAS Institute Inc., Cary, NC, USA).

\section{Results}

Out of 122 patients diagnosed with DLBCL during the pre-specified period, $96(74.8 \%)$ had a SEP before any chemotherapy, and among them, 12 (12.5\%; 8 males; median age: 68 [55-89] years) had L-TGL. Demographical and biological data for both the L-TGL ( $\mathrm{n}=12$; TGL[?] $5.5 \mathrm{~g} / \mathrm{L})$ and H-TGL ( $=84$; TGL>5.5 $\mathrm{g} / \mathrm{L})$ subgroups are summarized in Table 1.

Ann Arbor staging and International prognosis index were comparable between L-TGL and H-TGL groups. However no Richter syndrome was reported in the L-TGL group, but 2 were described in the H-TGL group. 


\section{IgG level was lower in L-TGL regardless of albumin and inflammatory status}

Immunoglobulin isotype levels were available only in two-thirds (8/12) of L-TGL and in approximately half $(46 / 84 ; 55 \%)$ of H-TGL patients, respectively: the median levels of IgG were significantly lower $(\mathrm{p}<0.001)$ in the L-TGL subgroup than in the H-TGL subgroup and in parallel with TGL levels, whereas the IgA and IgM levels were not significantly different $(\mathrm{p}=0.31$ and 0.32 , respectively). No patient had detectable viral load for Epstein Barr virus or Cytomegalovirus or active bacterial infection at diagnosis.

The serum total protein level was lower $(\mathrm{p}<0.01)$ and AG ratio was higher $(\mathrm{p}<0.01)$ in L-TGL than in H-TGL patients. Even though higher serum ferritin levels were found in L-TGL patients than in H-TGL patients $(\mathrm{p}=0.02)$, which could indicate higher biological inflammatory status or specific organ involvement, the levels of CRP were comparable in both subgroups.

Lymphocyte level was significantly lower in L-TGL patients $(\mathrm{p}=0.02)$, but was comparable between L-TGL and H-TGL deceased patients (Table 1).

\section{Death rate and infection related death are higher in L-TGL subgroup}

The mortality rate was higher in L-TGL (mortality rate: 10/12 [83\%] versus 22/84 [26.2\%]; $\mathrm{p}=0.03$ ) and median follow-up duration was shorter (follow-up duration: 15.2 months versus 55.53 months; $\mathrm{p}<0.001$ ) than in H-TGL subgroup (Table 1). Similarly, the rate of death caused by an infection was significantly higher in L-TGL than in H-TGL patients $(10 / 10$ [100\%] versus 6/22 [27.3\%]; $\mathrm{p}<0.001)$, as shown in Table 1. In H-TGL patients, deaths were caused by DLBCL progression for most patients $(54.5 \% ; 12 / 22$ deceased patients), whereas the remaining patients $(18.2 \% ; 4 / 22$ deceased patients) died from other causes that were independent of the background disease or related treatments.

Regarding infection-related deaths, no opportunistic infection was identified. All 10 deaths in L-TGL subgroup and 3 out of 6 infection-related deaths in H-TGL subgroups were related to pleuro-pneumopathy, either associated or not with ear, nose and throat infections and/or Streptococcus pneumoniae. The remaining 3 H-TGL infection-related deaths were caused by septic shock-complicated pyelonephritis, staphylococcus bacteremia and cutaneous cellulitis of the diabetic foot.

As seen on Kaplan Meier curves shown in Figure 1, the survival of L-TGL patients was significantly lower $(\mathrm{p}<0.001)$ than all the other groups considered in the whole DLBCL patients cohorts, including H-TGL patients $(\mathrm{n}=84)$, all patients who had a SEP (i.e. L-TGL and H-TGL patients considered together; $\mathrm{n}=96$ ) and patients who didn't get a SEP at diagnosis $(n=26)$.

\section{No concordance between TGL and albumin levels in L-TGL and H-TGL sub- groups}

In addition to the comparisons of demographics, clinical and biological characteristics in both L-TGL and HTGL subgroups, shown in Table 1; both subgroups were similar for all clinical and usual biological parameters including albumin level, except for the lower median TGL $(\mathrm{p}<0.01)$ and IgG levels $(\mathrm{p}<0.01)$ in L-TGL patients than in H-TGL patients, as expected. Moreover the AG ratio was higher in L-TGL than in H-TGL patients $(\mathrm{p}<0.01)$ indicating that TGL decrease was more pronounced than that of albumin, suggesting that albumin serum level was unrelated to TGL in our DLBCL patients subgroups.

\section{Chemotherapy regimen was comparable between L-TGL and H-TGL subgroups}

The distribution of chemotherapy regimens is shown in Table 2 between deceased and living patients in both the L-TGL and H-TGL subgroups. First-line chemotherapy was the R-CHOP regimen (21-day interval; rituximab, cyclophosphamide, hydroxyadriamycin, vincristine, and oral prednisone) for 85 (88.5\%) patients in total, whereas 3 others, all in the H-TGL subgroup, received ACBVP (two-week interval; adriamycin, 
cyclophosphamide, bleomycin, vindesine, and oral prednisone) followed by sequential consolidation therapy consisting of two cycles of methotrexate because of their younger age; 7 others ( 2 and 5 in the L-TGL and H-TGL subgroups, respectively) received the RMPV regimen (rituximab, methotrexate, procarbazine, and vincristine) because of cerebral involvement, and finally, the remaining patient was an elderly patient in the H-TGL subgroup who refused any chemotherapy.

A second-line chemotherapy regimen was chosen for 37 of the 96 patients, including 9 and 28 patients in the L-TGL and H-TGL subgroups respectively, whereas third- and fourth-line chemotherapy regimens were proposed for 10 patients and 2 patients, respectively. As shown in Table 2, the characteristics of patients who had died and those who remained alive at last follow-up in each subgroup appeared comparable, except for lymphoma stage: stage I lymphomas were significantly overrepresented in H-TGL patients who remained alive $(\mathrm{p}=0.02$; Table 2). Of note, stage IV lymphoma and central nervous system involvement were not significantly associated with death among H-TGL patients, although the low number of events precluded any firm conclusion (Table 2).

\section{Hypogammaglobulinemia at diagnosis is associated with higher risk of death}

Table 3 represents hazard ratio for the different variables studied using a cox proportional hazards model. Ann Arbor stage IV was positively associated with the occurrence of death (HR 3.47 (1.61-7.47), p < 0.01). More interestingly, L-TGL was also found to be independently associated with a higher risk of death (HR 12.8 [4.93-34.31], p <0.001) while H-TGL was associated with a lower risk of death (HR 0.21 [0.09-0.46], p $<0.01)$.

\section{Discussion}

This study is the first to estimate and emphasize on the frequency (12.5\%) and the clinical utility of LTGL determination at DLBCL diagnosis using systematic SEP. We demonstrated that L-TGL has a strong negative impact on the overall and infectious-related mortality rates. The higher AG ratio in L-TGL than in H-TGL patients suggests that the pronounced decrease in TGL levels and the associated clinical outcomes in the L-TGL subgroup may be 1) first, independent of the other possible associated unspecific causes and 2) second, mostly and directly related to one of our two hypotheses: either a pre-existent but previously undiagnosed PID on which DLBCL developed or an SID directly induced by the DLBCL condition. Finally, for both hypotheses, considered alone or in combination, the ability of a chemotherapy-induced SID to worsen, or reveal a greater susceptibility to infections in L-TGL patients is probable but remains unquantifiable in the context of the design of the present study. Our population was close to what has been described in France during this period of time, with a median age of 69 years, representing 35 to $40 \%$ of patients with lymphoma. $(23,24)$

At DLBCL diagnosis SEP is not unanimously recommended according to the international workgroup guidelines for malignant lymphoma(13-15), however from the results in our study we propose SEP to be performed at diagnosis. In our study, there were no significant differences between the two subgroups regarding inflammatory parameters, as assessed using CRP level, and albuminemia, suggesting that the decrease in TGL may not be secondary to metabolic/inflammatory causes or to increased loss of immunoglobulins but is probably directly related to low gamma-globulin production. In the same manner, since neither the Ann Arbor staging classification, IPI, nor the chemotherapy regimen were significantly different between the two groups, the increase in the overall and specific infection-related death rates may be attributed to the decrease in TGL in L-TGL subgroup. We didn't exclude neither Richter syndrome nor associated CNS involvement DLBCL subtypes that could exhibited worst malignant prognosis, because the first were identified only in H-TGL subgroup and both subtypes didn't show overall highest mortality rate in this study. Therefore, we may consider that this study drew up an overall picture of all subtypes of DLBCL. Moreover, showing that L-TGL patients exhibited a lower survival rate than all other possible subgroups of DLBCL, including patients who did not get a SEP at diagnosis, we further confirmed the importance of TGL assessment.

The main limitations of this study include its retrospective design with some missing data, including mainly 
immunoglobulin isotype levels, absence of systematic and reliable assessment of previous infections, and monitoring of TGL in the follow-up for the entire cohort. Consequently, two main secondary objectives could not be reached in this study: determination of an approach for eventual CVID diagnosis criteria and a differentiation scheme for the two causes of L-TGL considered herein, namely, primary or DLBCLrelated secondary immunodeficiencies. We assumed that combining analyses of both DLBCL and TGL outcomes under chemotherapy should partially help to differentiate these two causes of decrease in TGL for each patient. Indeed, complete or partial remission of DLBCL and concomitant increase in TGL under chemotherapy would support a DLBCL-related SID. Conversely, in cases of a lack of response or early death, no conclusion could be drawn, since chemotherapy would induce a further decrease in TGL for an unknown duration.

To conclude, while epidemiological data on lymphoma treatment strategies are optimistic and have seen improvements in survival throughout the years. This study, using a simple, widely and easily available biological test, seems to show that L-TGL patient subgroup exhibits worse prognosis with higher overall and infection-related mortality risks than H-TGL subgroup. Therefore, this study argues for the need to systematically perform at least SEP at lymphoma and especially at DLBCL diagnosis and emphasizes on the need of further studies to confirm these results especially in prospective studies: 1) to determine nosological and prognostic distinctions between possible previously undiagnosed PIDs and DLBCL-related SIDs using, for example, next-generation sequencing methods to uncover molecular defects of humoral PIDs(22,25) and to assess the timing of a possible TGL recovery after chemotherapy in relation to lymphoma outcomes; 2) to assess the possible clinical benefit of immunoglobulin supplementation for SIDs, or prophylactic antibiotics, taking into account advances in chemotherapy and management strategies in Onco-Haematology.

\section{References}

1. Cunningham-Rundles C, Siegal FP, Cunningham-Rundles S, Lieberman P. Incidence of cancer in 98 patients with common varied immunodeficiency. J Clin Immunol. 1987 Jul;7(4):294-9.

2. Rezaei N, Hedayat M, Aghamohammadi A, Nichols KE. Primary immunodeficiency diseases associated with increased susceptibility to viral infections and malignancies. J Allergy Clin Immunol. 2011 Jun;127(6):1329-1341.e2; quiz 1342-3.

3. Baldovino S, Montin D, Martino S, Sciascia S, Menegatti E, Roccatello D. Common variable immunodeficiency: crossroads between infections, inflammation and autoimmunity. Autoimmun Rev. 2013 Jun;12(8):796-801.

4. Vajdic CM, Mao L, van Leeuwen MT, Kirkpatrick P, Grulich AE, Riminton S. Are antibody deficiency disorders associated with a narrower range of cancers than other forms of immunodeficiency? Blood. 2010 Aug 26;116(8):1228-34.

5. Shapiro RS. Malignancies in the setting of primary immunodeficiency: Implications for hematologists/oncologists. Am J Hematol. 2011 Jan;86(1):48-55.

6. Tran H, Nourse J, Hall S, Green M, Griffiths L, Gandhi MK. Immunodeficiency-associated lymphomas. Blood Rev. 2008 Sep;22(5):261-81.

7. Mellemkjaer L, Hammarstrom L, Andersen V, Yuen J, Heilmann C, Barington T, et al. Cancer risk among patients with IgA deficiency or common variable immunodeficiency and their relatives: a combined Danish and Swedish study. Clin Exp Immunol. 2002;130(3):495-500.

8. Oksenhendler E, Gerard L, Fieschi C, Malphettes M, Mouillot G, Jaussaud R, et al. Infections in 252 patients with common variable immunodeficiency. Clin Infect Dis Off Publ Infect Dis Soc Am. 2008 May 15;46(10):1547-54.

9. Cunningham-Rundles C. The many faces of common variable immunodeficiency. Hematol Am Soc Hematol Educ Program. 2012;2012:301-5. 
10. CEREDIH: The French PID study group. The French national registry of primary immunodeficiency diseases. Clin Immunol Orlando Fla. 2010 May;135(2):264-72.

11. Picard C, Al-Herz W, Bousfiha A, Casanova J-L, Chatila T, Conley ME, et al. Primary Immunodeficiency Diseases: an Update on the Classification from the International Union of Immunological Societies Expert Committee for Primary Immunodeficiency 2015. J Clin Immunol. 2015 Nov;35(8):696-726.

12. Chan H-Y, Yang Y-H, Yu H-H, Chien Y-H, Chiang L-L, Chiang B-L. Clinical characteristics and outcomes of primary antibody deficiency: a 20-year follow-up study. J Formos Med Assoc Taiwan Yi Zhi. 2014 Jun;113(6):340-8.

13. Dabaja BS, Advani R, Hodgson DC, Dhakal S, Flowers CR, Ha CS, et al. ACR Appropriateness Criteria(r) Diffuse Large B-Cell Lymphoma: Am J Clin Oncol. 2015 Dec;38(6):610-20.

14. Horwitz SM, Zelenetz AD, Gordon LI, Wierda WG, Abramson JS, Advani RH, et al. NCCN Guidelines Insights: Non-Hodgkin's Lymphomas, Version 3.2016. J Natl Compr Cancer Netw JNCCN. 2016;14(9):106779 .

15. Tilly H, Gomes da Silva M, Vitolo U, Jack A, Meignan M, Lopez-Guillermo A, et al. Diffuse large B-cell lymphoma (DLBCL): ESMO Clinical Practice Guidelines for diagnosis, treatment and follow-up. Ann Oncol. 2015 Sep;26:v116-25.

16. Raanani P, Gafter-Gvili A, Paul M, Ben-Bassat I, Leibovici L, Shpilberg O. Immunoglobulin prophylaxis in chronic lymphocytic leukemia and multiple myeloma: systematic review and meta-analysis. Leuk Lymphoma. 2009 May;50(5):764-72.

17. Jolles S, Chapel H, Litzman J. When to initiate immunoglobulin replacement therapy (IGRT) in antibody deficiency: a practical approach: When to initiate IgG therapy in antibody deficiency. Clin Exp Immunol. 2017 Jun;188(3):333-41.

18. Patel SY, Carbone J, Jolles S. The Expanding Field of Secondary Antibody Deficiency: Causes, Diagnosis, and Management. Front Immunol [Internet]. 2019 Feb 8 [cited 2020 Aug 24];10. Available from: https://www.frontiersin.org/article/10.3389/fimmu.2019.00033/full

19. Benbrahim O, Viallard J-F, Choquet S, Royer B, Bauduer F, Decaux O, et al. A French observational study describing the use of human polyvalent immunoglobulins in hematological malignancy-associated secondary immunodeficiency. Eur J Haematol. 2018 Jul;101(1):48-56.

20. Seidel MG, Kindle G, Gathmann B, Quinti I, Buckland M, van Montfrans J, et al. The European Society for Immunodeficiencies (ESID) Registry Working Definitions for the Clinical Diagnosis of Inborn Errors of Immunity. J Allergy Clin Immunol Pract. 2019 Aug;7(6):1763-70.

21. Ameratunga R, Woon S-T, Gillis D, Koopmans W, Steele R. New diagnostic criteria for common variable immune deficiency (CVID), which may assist with decisions to treat with intravenous or subcutaneous immunoglobulin. Clin Exp Immunol. 2013 Nov;174(2):203-11.

22. Ameratunga R, Woon S-T. Perspective: Evolving Concepts in the Diagnosis and Understanding of Common Variable Immunodeficiency Disorders (CVID). Clin Rev Allergy Immunol. 2020 Aug;59(1):109-21.

23. Perry AM, Diebold J, Nathwani BN, MacLennan KA, Muller-Hermelink HK, Bast M, et al. Non-Hodgkin lymphoma in the developing world: review of 4539 cases from the International Non-Hodgkin Lymphoma Classification Project. Haematologica. 2016 Oct;101(10):1244-50.

24. Desandes E, Lacour B, Clavel J, le Reseau francais des registres de cancers. [Cancer in adolescents and young adults in France: Epidemiology and pathways of care]. Bull Cancer (Paris). 2016 Dec;103(12):957-65.

25. Mahlaoui N, Picard C, Bach P, Costes L, Courteille V, Ranohavimparany A, et al. Genetic diagnosis of primary immunodeficiencies: A survey of the French national registry. J Allergy Clin Immunol. 2019 Apr;143(4):1646-1649.e10. 
Figure and Tables

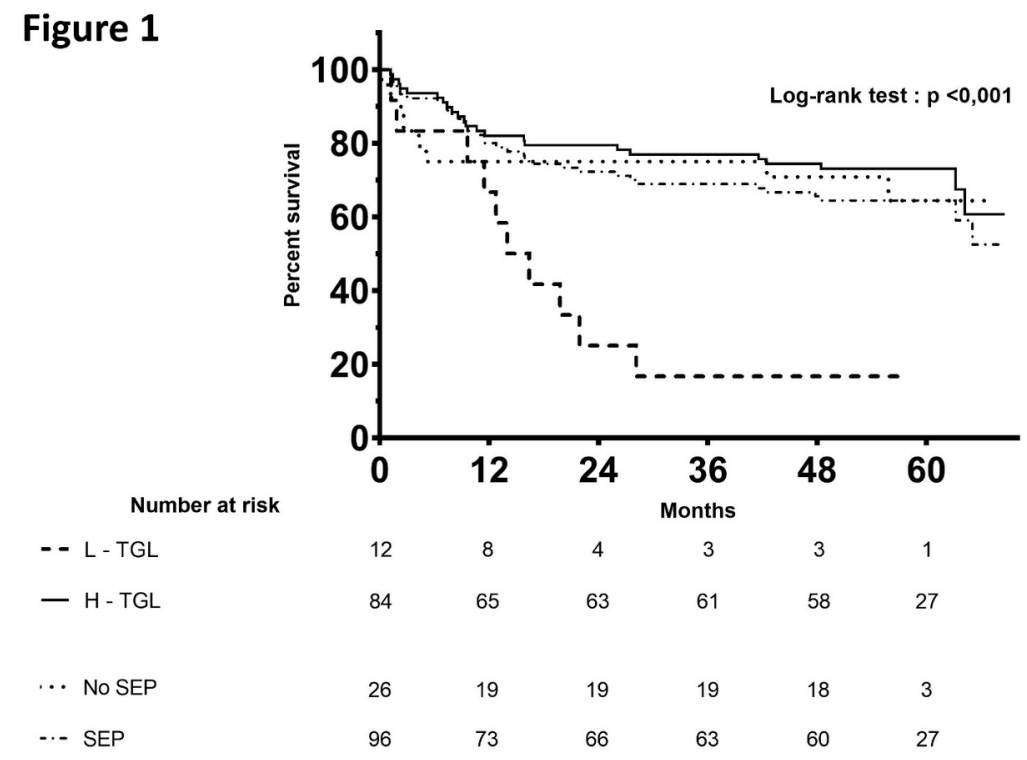

Figure 1: Kaplan-Meier survival curves of diffuse large B cell lymphoma patients according to serum total gamma-globulin levels (TGL; lowest (L-) or highest (H-)TGL)

L-TGL: The Lowest-TGL; H-TGL: The Highest-TGL.SEP: Patients with serum electrophoresis; No SEP:Patients without SEP at diagnosis. Time is expressed in months on the $\mathrm{X}$-axis.

Table I: Diffuse Large B-cell Lymphoma patients' characteristics and outcome according to serum total gammaglobulin levels (TGL; lowest (L-) or highest (H-) TGL)

\begin{tabular}{lll}
\hline & All patients & All patients \\
\hline & L-TGL $([?] 5.5 \mathrm{~g} / \mathrm{L})$ patients & H-TGL $(>5.5 \mathrm{~g} / \mathrm{L})$ patient \\
DLBCL $(\mathrm{n}=)$ & 12 & 84 \\
Sex $(\mathrm{M} / \mathrm{F})$ & $7 / 5$ & $33 / 51$ \\
Age (years) median [range] & $65[55-80]$ & $70[21-89]$ \\
Lymphoma Ann-Arbor-Stage & Lymphoma Ann-Arbor-Stage & Lymphoma Ann-Arbor-Stag \\
I & 0 & 16 \\
II & 1 & 15 \\
III & 4 & 15 \\
IV & 7 & 38 \\
Central nervous system involvement (CNS) & 2 & 5 \\
Richter syndrome (RS) & 0 & 2 \\
International Prognostic Index (IPI)/Risk group (n=) & $10(\mathrm{CNS}$ excluded) \\
(0-1) Low & 1 & 77 (CNS and RS excluded) \\
(2) Low-Intermediate & 2 & 7 \\
(3) High-Intermediate & 4 & 28 \\
(4-5) High & 4 & 19 \\
Total gammaglobulin level (g/L) median [range] & $4.8[2.7-5.5]$ & 23 \\
Immunoglobulin isotype levels dosage, (n=) & 8 & $9.15[5.9-19.3]$ \\
& & 46
\end{tabular}




\begin{tabular}{lll}
\hline & All patients & All patients \\
\hline IgG level (g/L) median [range] & $5.4[2.8-5.45]$ & $9.6[6.4-15.7]$ \\
IgA level (g/L) median [range] & $0.7[0.35-5.91]$ & $2.3[0.3-5.0]$ \\
IgM level (g/L) median [range] & $0.4[0.2-3.7]$ & $1.0[0.2-3.3]$ \\
CRP level (g/L) median [range] & $31[3-116]$ & $15[3-237]$ \\
Ferritin level ( $\mu \mathrm{g} / \mathrm{L})$ median [range] & $433[73-1628]$ & $170[4-3472]$ \\
Albumin level (g/L) median [range] & $32.5[24-40.7]$ & $39[16-50]$ \\
Serum Total protein level (g/L) median [range] & $58.17[46-90]$ & $64.5[46-72]$ \\
AG-Ratio median [range] & $6.79[4.62-12.22]$ & $4.39[1.7-7.13]$ \\
LDH (Unit/L) median [range] & $341.5[156-2784]$ & $278[132-10091]$ \\
Lymphocytes count (G/L) median [range] & $0.8[0.2-2.58]$ & $1.26[0.18-7.56]$ \\
Follow-up (months), median [range] & $15.2[1.23-57.77]$ & $55.53[1.17-68.6]$ \\
Deaths & $10(83.3 \%)$ & $22(26.2 \%)$ \\
Causes of deaths Infections DLBCL progression Other causes & $10(100 \%) 0(0 \%) 0(0 \%)$ & $6(27.3 \%) 12(54.5 \%) 4(18 \%$ \\
\hline
\end{tabular}

TGL : Total serum Gammaglobulin Level; L-TGL : Lowest-TGL; H-TGL : Highest-TGL; NA: not applicable;IgG (or A, or M) : serum dosage of Immunoglobulin G (or A or M); CRP : serum C-Reactive Protein; AG-Ratio : Ratio of serum Albumin level on Total serum Gammaglobulin level; DLBCL:Diffuse-LargeB-Cell-Lymphoma

Table II: Demographical, clinical and main biological characteristics in Diffuse-Large-B-CellLymphoma deceased patients and patients alive, according to serum total gammaglobulin levels (TGL; lowest (L-) or highest (H-)TGL)

\begin{tabular}{lll}
\hline & L-TGL (TGL [?] 5.5 g/L) patients & L-TGL (TGL [?] 5.5 g/L) pat \\
\hline & Deceased Patients & Patients alive \\
$\mathrm{N}=$ & 10 & 2 \\
Sex (M/F) & $6 / 4$ & $1 / 1$ \\
Age (years) median [range] & $65[57-80]$ & $66[55-78]$ \\
Lymphoma Ann-Arbor-Stage & & \\
I & 0 & 0 \\
II & 1 & 0 \\
III & 2 & 2 \\
IV & 7 & 0 \\
Central nervous system involvement (CNS) & 2 & 0 \\
Richter syndrome (RS) & 0 & 0 \\
International Prognostic Index (IPI)/Risk group (n=) & $8(\mathrm{CNS}$ excluded) \\
(0-1) Low & 1 & 2 \\
(2) Low-Intermediate & 1 & 0 \\
(3) High-Intermediate & 2 & 0 \\
(4-5) High & 4 & 2 \\
Chemotherapy Regimen & & 0 \\
First-line & & \\
RCHOP & $8(80 \%)$ & $2(100 \%)$ \\
RMPV & $2(20 \%)$ & 0 \\
ABVD & 0 & 0 \\
Second-line & $7(70 \%)$ & $2(100 \%)$ \\
Third-line & $3(30 \%)$ & 0 \\
Fourth-line & $1(10 \%)$ & 0 \\
Total gammaglobulin level (g/L) median [range] & $5.2[2.7-5.5]$ & $4.15[3.8-4.5]$ \\
& &
\end{tabular}




\begin{tabular}{|c|c|c|c|c|}
\hline & & L-TGL (TGL [?] 5.5 & atients & L-TGL (TGL [?] \\
\hline Immunoglobulin isotype le & els, $n$ & 7 & & 1 \\
\hline IgG level $(\mathrm{g} / \mathrm{L})$ median $[\mathrm{ra}$ & ige] & $5.45[2.8-5.45]$ & & 4.44 \\
\hline IgA level $(\mathrm{g} / \mathrm{L})$ median $[\mathrm{ra}$ & ge] & $0.61[0.35-5.91]$ & & 1.11 \\
\hline IgM level $(\mathrm{g} / \mathrm{L})$ median $[\mathrm{ra}$ & age] & $0.46[0.2-3.7]$ & & 0.2 \\
\hline CRP level $(\mathrm{g} / \mathrm{L})$ median $[\mathrm{r}$ & inge] & $26.5[3-116]$ & & 65 \\
\hline Ferritin level $(\mu \mathrm{g} / \mathrm{L})$ media & a [range] & $538.5[73-1628]$ & & 112 \\
\hline Albumin level $(\mathrm{g} / \mathrm{L})$ media & [range] & $33.8[24-40.7]$ & & 32 \\
\hline AG-Ratio median [range] & & $6.79[4.62-12.22]$ & & 7.63 \\
\hline LDH (Unit/L) median [rar & & $285[156-2784]$ & & 776.5 [537-1016] \\
\hline Lymphocytes count $(\mathrm{G} / \mathrm{L})$ & median [range] & $0.8[0.2-2.58]$ & & $0.62[0.29-0.95]$ \\
\hline $\begin{array}{l}\text { TGL : Total serum Gamm } \\
\text { M) : serum dosage of Imm } \\
\text { of serum Albumin level on }\end{array}$ & $\begin{array}{l}\text { aglobulin Level; L-TGL } \\
\text { noglobulin G (or A or M) } \\
\text { Total serum Gammaglob }\end{array}$ & $\begin{array}{l}\text { owest-TGL; H-TGI } \\
\text { RP : serum C-Reac } \\
\text { level }\end{array}$ & $\begin{array}{l}\text { st-TGL } \\
\text { ein; AG }\end{array}$ & $\begin{array}{l}\text { gG (or A, or } \\
\text { Ratio : Ratio }\end{array}$ \\
\hline $\begin{array}{l}\text { Table III: Univariate a } \\
\text { factors associated with }\end{array}$ & $\begin{array}{l}\text { nd multivariate Cox } \\
\text { the occurrence of deat }\end{array}$ & $\begin{array}{l}\text { portional hazards } \\
\text { n DLBCL }\end{array}$ & analys & is examining \\
\hline & Hazard ratios $(95 \% \mathrm{CI})$ & Univariate $\mathrm{p}$ value & Multiva & iriate $\mathrm{p}$ value \\
\hline Sexe & $1.4[0.56-3.7]$ & 0.45 & & \\
\hline L-TGL & $12.8[4.93-34.31]$ & $<0.001$ & $<0.01$ & \\
\hline H-TGL & $0.21[0.09-0.46]$ & $<0.01$ & - & \\
\hline $\begin{array}{l}\text { Inflammation }(\mathrm{CRP}>10 \\
\mathrm{mg} / \mathrm{L})\end{array}$ & $5.5[1.54-35.2]$ & $<0.01$ & NS & \\
\hline $\begin{array}{l}\text { Hyperferrininemia } \\
\text { (Ferritine > } 300 \mathrm{ug} / \mathrm{L})\end{array}$ & $6.22[1.5-41.9]$ & 0.01 & NS & \\
\hline Stage IV & $4.5[1.6-16.02]$ & $<0.01$ & NS & \\
\hline Cerebral & $1.5[0.24-5.42]$ & 0.59 & & \\
\hline Richter & $4.16[0.23-20.34]$ & 0.25 & & \\
\hline Second line treatment & $4.32[1.6-13.5]$ & $<0.01$ & NS & \\
\hline Third line treatment & $1.76[0.41-5.35]$ & 0.40 & & \\
\hline
\end{tabular}

HR: Hazard ratio; TGL : Total serum Gammaglobulin Level; L-TGL : Lowest-TGL; H-TGL : HighestTGL; 